\title{
Recombinase Polymerase Amplification (RPA) an isothermal technique suitable for a range of applications- Evaluation of RPA volume tolerance
}

\section{Overview}

DNA/RNA amplification performed by PCR or isothermal methods operating above $50^{\circ} \mathrm{C}$ is often restricted to reaction volumes in the range of 10-100 $\mu$ l, and may require oil overlays or similar containment strategies. This is because, on the one hand, evaporation can limit small reaction volume formats, while on the other hand use of larger reaction volumes can complicate the consumable design required for efficient thermal control during temperature cycling events (or if isothermal, require longer to reach temperature). TwistDx's proprietary Recombinase Polymerase Amplification (RPA) method addresses these challenges. The low running temperature (typically $37-42^{\circ} \mathrm{C}$, can be less) and rapid amplification time (typically 3-15 minutes) reduces problems caused by evaporation even without oil overlay or other containment approaches. Similarly large reaction volumes are easily deployed because the combination of low temperature operation and, more critically, resistance to background noise accumulation at lower temperatures, mean that delay in reaching optimal operating temperature is not detrimental to performance. Consequently the RPA technology offers key advantages for applications occurring in both the microfluidic domain (for example digital approaches) as well as applications which may benefit from larger "macro" volume, for example low complexity molecular tests in which simple biological sample dilution is employed. In this application note we demonstrate RPA performance in reactions established in quite different total reaction volumes and performed in standard PCR tubes (0.2ml) without oil overlays or any other specialised consumables.

\section{Materials and Methods}

TwistAmp ${ }^{\circledR}$ Basic kit (50 $\mu$ l) reactions were prepared as follows:

- $\quad$ Each freeze-dried pellet was rehydrated with $29.5 \mu \mathrm{l}$ rehydration buffer, $7 \mu \mathrm{l}$ primer mix (Basic kit positive control), $8.5 \mu \mathrm{l} \mathrm{dH} 20,4 \mu \mathrm{l}$ magnesium acetate $(280 \mathrm{mM}), 1 \mu \mathrm{l}$ of positive control template (250 copies/ul) or $1 \mu \mathrm{l} \mathrm{dH2O} \mathrm{for} \mathrm{no} \mathrm{template}$ control (ntc). For a 100 and 200ul volume reaction two and four $50 \mu \mathrm{l}$ pellets were pooled respectively and rehydrated with the required multiple of reagents, for 10,5 and $2.5 \mu \mathrm{l}$ volumes a $50 \mu \mathrm{l}$ reaction was split to provide the smaller volumes.

- Reactions were incubated for an hour at a temperature of $40^{\circ} \mathrm{C}$ in a heat block; reactions were briefly agitated after 4

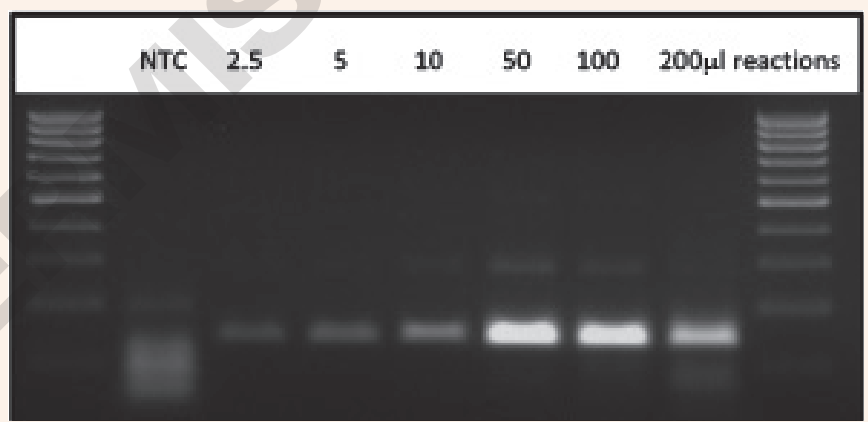

Figure 1. DNA amplification using TwistAmp ${ }^{\circledR}$ Basic reactions (250 copies positive control) at incubation temperature of $40 \mathrm{oC}, 2 \%$ agarose gel, * $100 \%$ of all samples loaded except $100 / 200 \mathrm{ml}$ reactions where $50 \mu \mathrm{l}$ was cleaned up and ran on gels. No template control (NTC) was a $50 \mu$ reaction. minutes incubation.

- $\quad$ Post amplification, reactions were cleaned with a standard PCR clean-up kit and run on a $2 \%$ agarose gel (50 $\mu$ only for the larger reactions).

\section{Summary}

All reaction volumes amplified a correctly sized product. This flexible reaction size provides evidence of RPA suitability for a multitude of applications. Currently TwistAmp ${ }^{\circledR}$ users are applying the technology in small volumes such as droplets in conjunction with electrowetting on a microfluidic platform with single copy detection within 15 minutes (1). Others have used droplets with a SlipChip platform (2\&3). Centrifugal step emulsification has also been successfully utilised (4), as have other oil water emulsion methods (5). Large reaction volume use has yet to be exploited, but potentially could benefit industries such as food testing where inhibitors and low titre samples can be problematic.

The whole range of TwistAmp ${ }^{\circledR}$ products can be purchased atwww.twistdx.co.uk/products or over the phone at $+44(0) 1223496700$

\section{References}

(1) http://www.ncbi.nlm.nih.gov/pubmed/26086197 (2) http://pubs.acs.org/doi/abs/10.1021/ac200247e

(3) http://www.ncbi.nlm.nih.gov/pubmed/27074005 (4) http://pubs.rsc.org/en/content/articlehtml/2015/lc/c5lc00291e

(5) http://www.ncbi.nlm.nih.gov/pubmed/?term=24070333\%5Buid\%5 